Macedonian Pharmaceutical Bulletin, 66 (Suppl 1) 229 - 230 (2020)

Online ISSN $1857-8969$

UDC: 616-036.12-051:615.15(497.7)

DOI: 10.33320/maced.pharm.bull.2020.66.03.114

Short communication

\title{
Community pharmacists' attitudes toward the chronic disease management in R.N. Macedonia - part II
}

\author{
Donka Pankov*, Nikola Geskovski, Maja Simonoska Crcarevska, Marija Glavas Dodov \\ Institute of pharmaceutical technology, Faculty of Pharmacy, Ss. Cyril and Methodius University, \\ Majka Tereza 47, 1000 Skopje, N. Macedonia
}

\section{Introduction}

Worldwide patients tend to consult pharmacists as a first call for advice, not just for their medication therapy but also for the health problems. Community pharmacists are increasingly expected to improve disease management by aiming to improve the effective drug therapy, reducing drug-drug interactions and drug-related side effects. Creating personal patient relationships and fostering communication can help to improve medication adherence and make chronic disease management more successful (Cheema, 2015).

Patient engagement is one of the best tools in a community pharmacist's arsenal in handling chronic disease management. Making sure patients are involved in and knowledgeable about their health is vital when addressing a chronic disease. Namely, when patients are fully engaged in their care, they are more likely to maintain treatment plans, follow their health, and ask questions about their illness or life-style protocols (Krist et al., 2020).

Therefore, the aim of the present study was to investigate community pharmacists' attitudes, perceptions, practice and perceived barriers in ensuring the proper pharmaceutical care for patients with chronic diseases in the Republic of North Macedonia (R.N.M).

*dpankov0@gmail.com

\section{Materials and methods}

For the purpose of this survey, a structured pretested (Pankov, 2016) questionnaire was used. The questionnaire included 13 items related to the community pharmacists` attitudes and perceptions in everyday practice with chronic disease patients as well as their knowledge for chronic disease management.

The responses were collected between September $1^{\text {st }} 2016$ and February 28th, 2017.

Obtained data were tabulated using Microsoft Excel® (Microsoft Corp. Redmond, WA, USA), computed and consequently evaluated using statistical software STAT-GRAPHICS Centurion XVI evaluation (Stat Point technologies Inc., USA).

\section{Results and discussion}

The study is based on responds of 310 community pharmacists of which $82.58 \%$ were females. The average age was $38.83 \pm 9.8$ years. Majority of pharmacists, $57.42 \%$ think that patients with chronic diseases are not well educated to the level where they can successfully manage their disease, while $36.13 \%$ think that patients are educated, but not all of them.

A vast majority of $80 \%$ agreed that with the extension of pharmaceutical care and education of patients with chronic diseases, use of prescribed 
drugs will be more clarified to the patients and their compliance will be improved. Similar percentage of pharmacists $(\sim 83 \%)$ responded affirmatively to the possibility of augmentation of health care services and their active engagement in the care and prevention of patients with chronic diseases.

Nearly $75 \%$ of respondents think that through the active involvement of pharmacists in the education of chronic patients how to use correctly medical devices (inhalers, insulin pens, or medical devices to control blood pressure, etc.) patients will be able to use them properly and will successfully manage their therapy as well as that the outcome of the therapy will improve.

Almost half of the pharmacists (57.42\%) agreed that the pharmacist, as the most accessible health worker, can be actively involved in the community pharmacy setting in measuring blood pressure, glucose and blood cholesterol, etc. However, $25.81 \%$ do not agree with these activities as they think that there are no conditions in the setting and $16.77 \%$ think that they should not be conducted by a pharmacist. Also, $77.42 \%$ believe that the pharmacists can contribute to the prevention of chronic diseases through life-changing services such as weight regulation, smoking cessation and alcohol consumption, assessment of risk factors for certain chronic diseases, while $9.35 \%$ do not agree with previous.

$84.52 \%$ think it is necessary for pharmacists to inform medical doctors if they are carrying out additional pharmaceutical care in order to achieve better collaboration.

Faculty of pharmacy, UKIM - Skopje and University clinics are most trusted among pharmacists $(80.32 \%)$ when it comes to continuous education related to pharmaceutical care in the prevention and care of patients with chronic diseases. They are followed by pharmaceutical companies (12.9\%) and internet education $6.77 \%$.

$47.42 \%$ noted that their pharmacy can provide place/space for the chronic disease patients' education/consultation, while $21.61 \%$ responded that it is not possible. In the context, majority $(77.1 \%)$ responded that are interested in providing additional pharmaceutical care to patients with chronic diseases if the adequate conditions are fulfilled and only $3.87 \%$ are not interested. Nevertheless, $48.06 \%$ think that they have appropriate level of knowledge for the provision of education/pharmaceutical care of patients with chronic disease and similar percentage $(48.06 \%)$ stated that will need additional training. Merely, $4.52 \%$ and $0.97 \%$ noted that they do not know or are not ready, consequently.

Immense majority $(83.23 \%)$ think that additional pharmaceutical care should be valued by extra reimbursement and only $11.61 \%$ think that it should be in the frame of usual pharmaceutical care i.e. without any supplementary reimbursement.

Opinion of $17.48 \%$ of respondents is that by additional pharmaceutical care in general health costs will be reduced as a result of prevention/delay of chronic diseases and their complications, $6.45 \%$ think that health services by other health professionals will be reduced and thus savings will be produced and majority $-69.33 \%$ agree with both. Only, $6.45 \%$ do not agree with either.

\section{Conclusion}

The results of the conducted study pointed that community pharmacists in the Republic of North Macedonia possess knowledge, possibility and willingness to implement additional pharmaceutical care for patients with chronic disease in the everyday community pharmacy practice. Also, they stated that such activities should be valued by added reimbursement. By provision of supplementary pharmaceutical care for patients with chronic diseases they agree that patient therapy management and compliance will be significantly improved and thus the outcome of the overall medical treatment will be more effective.

\section{References}

Cheema, E., 2015. The role of pharmacists in the management of chronic disease and prevention of adverse drug reactions (Doctoral dissertation, University of Warwick).

Krist, A.H., Tong, S.T., Aycock, R.A., Longo, D.R., 2017. Engaging patients in decision-making and behavior change to promote prevention. Studies in health technology and informatics 240, 284-302.

Pankov, D., Crcarevska, M.S., Mladenovska, K., Raicki, R.S. and Dodov, M.G., 2016. Role of community pharmacists in chronic disease management in the Republic of Macedonia. Mac. Pharm. Bull. 62 (suppl), 621-622.

Maced. Pharm. Bull. 66 (Suppl 1) 229 - 230 (2020) 